\title{
HETEROZIGOSE INDIVIDUAL E MATERNA SOBRE O GANHO DE PESO DO NASCIMENTO AO DESMAME DE TERNEIROS PAMPIANO-BRAFORD
}

\author{
INDIVIDUAL AND MATERNAL HETEROZIGOSIS ON PREWEANING \\ WEIGHT GAIN OF PAMPIANO-BRAFORD CALVES
}

\author{
Patricia Guidoux Leal Wolf ${ }^{1}$ Ricardo Macedo Gregory ${ }^{2}$ \\ Rodrigo Costa Mattos ${ }^{2}$ Fernanda Varnieri Brito ${ }^{3}$
}

RESUMO

Foram analisados dados de ganho de peso do nascimento ao desmame (GNDa) de animais Pampiano-Braford (cruzamento de Nelore X Hereford) criados no Rio Grande do Sul com a finalidade de verificar a influência da heterose individual (HI) e materna (HM) sobre o GNDa e quantificá-la. Os dados foram pré-ajustados para idade da vaca (IV), data juliana de nascimento (DJN) e idade do terneiro ao desmame (IT). Entretanto, análises exploratórias sugeriram que as pré-correções para IV, DJN e IT não estão ajustando corretamente os dados $(P<0,01)$ no período pré-desmame. Em função da estrutura $e$ conexidade do conjunto de dados, as análises foram realizadas de duas maneiras distintas. Em uma primeira etapa, estimou-se simultaneamente HI e HM a partir de um arquivo contendo 4186 animais (arquivo 2), obtendo-se valores de 31,91 e 33,53kg, respectivamente. Na segunda etapa, estimou-se HI a partir de um subconjunto do arquivo 2 (subconjunto 1) com 1507 registros com heterozigose materna 0,0000 e HM de outro subconjunto do arquivo 2 (subconjunto 2) com 2777 registros com heterozigose materna individual 0.5000; os valores estimados foram 13,79e $34,08 \mathrm{~kg}$, respectivamente. Todos os valores de heterose sobre o $G N D a$ encontrados foram significativos $(P<0,01)$, e a relação entre heterose e heterozigose para esta característica apresentou um comportamento linear $(P<0,01)$, considerando-se que o cruzamento estudado foi somente entre uma raça taurina e uma zebuína. Verificou-se que neste trabalho, os animais Hereford possuíam valor genético aditivo superior ao dos cruzados, todavia o mesmo não foi suficiente para contrabalançar os efeitos da heterose sobre o GNDa. Na comparação com os valores utilizados pelo Método GenSys, os valores de HI e HM, obtidos no presente trabalho, produziram diferenças na ordem de classificação de produtos ao desmame, mas o número de animais selecionados em comum pelos dois métodos não sofreu grandes mudanças. As vacas com composição racial 1/2 tiveram o melhor desempenho no GNDa, enquanto os terneiros com heterozigose individual máxima (composição racial 1/2) foram superados pelos 3/8 (filhos de vacas 1/2), quando se inclui a HM no desempenho dos mesmos, salientando a importância da HM sobre o GNDa.

Palavras-chave: gado de corte, reprodução animal, heterozigose, ganho de peso, desmame.

\section{SUMMARY}

Data on preweanning gain (GNDa) of PampianoBraford animals raised in Rio Grande do sul were analyzed to verify the influence of individual (HI) and maternal (HM) heterosis in GNDa, and to quantify it. Data were adjusted for dam age (IV), julian birth date (DJN)and calf age at weaning,(IT). However, exploratory analysis have suggested that the adjustment factors for IV, DJN, and IT are not doing this adjustment correctly $(P<0.01)$. Because of the structure and connectedness of the major data set, the analysis was done in two different ways. In the first phase, HI and HM were estimated simultaneously, in file 2, compose of 4186 animals, and the values obtained were 31.91 and $33.53 \mathrm{~kg}$, respectively. In the second phase, HI was estimated in a subset of file 2 (subset 1) compose of 1507 animals with maternal heterozigosis 0.0000; HM was estimated in subset 2 of file 2 with 2777 animals with individual heterozigozis 0.5000 . The values found were 13.79 and $34.08 \mathrm{~kg}$, respectively. All the values found for heterosis on GNDa were significant $(P<0.01)$, and the relation between heterosis and heterozigosis for this characteristic was linear $(P<0.01)$. It was show that the Hereford animals, in this study, had greater genetic additive values than crossbreeds, but this difference was not sufficient to supress the effects of heterosis on GNDa. In comparison with the values used in GenSys Method, the HI and $H M$ values, obtained in this study produced a change in the ranking of calves at preweaning, but the number of animals chosen in common by the two methods did not differ much. The cows with 1/2 breed composition had the best performance on

${ }^{1}$ Médico Veterinário, Mestre, Autônomo, Porto Alegre, RS.

${ }^{2}$ Médico Veterinário, Doutor, Professor Adjunto, Pesquisador CNPq, REPROLAB, Departamento. De Medicina Animal, Faculdade de

Veterinária, CP 15094, 91501-970 Porto Alegre, RS. Autor para correspondência. E-mail: hereford@ voyager.com.br

${ }^{3}$ Engenheiro Agrônomo, Mestre, GenSys Consultores Associados, Porto Alegre, RS.

Recebido para publicação em 28.08.98. Aprovado em 09.12.98 
GNDa, while the calves with maximum heterosis (breed composition 1/2) were excelled by those 3/8, when HM was included in the performance, emphasizing the importance of HM on GNDa.

Key words: beef cattle, animal reproduction, heterozigosis, weight gain, weaning.

\section{INTRODUÇÃO}

A raça Pampiano-Braford está crescendo de forma expressiva no Brasil. Como se trata de uma raça ainda em formação e produto de cruzamento entre raças (Hereford x Zebuínos - na maioria Nelore), deve-se estar atento para que não haja incremento somente em quantidade, priorizando sim, a qualidade dos mesmos. A heterose obtida em cruzamentos entre raças zebuínas e européias é consideralvelmente maior do que aquela fruto de cruzamentos entre raças européias, com grandes possibilidades para obter-se aumento da produtividade em gado de corte (CHAGAS, 1983). Considerando regiões de clima tropical e subtropical, CUNNINGHAM (1989) cita que o cruzamento entre raças zebuínas e européias gera linhagens com melhor produtividade. Nas últimas 4 décadas, o cruzamento entre raças de bovinos de corte tornou-se uma poderosa arma para os produtores aumentarem, de forma econômica, a produtividade de seus rebanhos. Este fato também ocorreu na suínocultura e na avicultura (PADILHA, 1994).

A heterozigose é um conceito estatístico que define qual a probabilidade dos alelos de um determinado "locus" provirem de raças distintas; já a heterose representa o fenômeno real que se verifica na produção animal ante a presença de heterozigose (FRIES, 1994). O sucesso de um cruzamento depende da escolha adequada da combinação das raças para determinado ambiente, manejo e sistema de produção, a fim de que haja suporte para o potencial de incremento na produção oferecido pelo gado cruzado (KOGER, 1980). Na escolha das raças envolvidas no cruzamento, LEAL (1993) avalia fatores de grande importância que influenciam a produtividade de uma operação pecuária, tais como: recurso genético animal e forrageiro; clima e demanda de mercado. FRIES (1996) analisou diferentes tipos de cruzamentos e demonstrou que, nos casos em que tanto raças taurinas como zebuínas participam na composição de cruzamentos, há um afastamento da linearidade na relação entre heterozigose e heterose.

Os objetivos do presente trabalho foram: verificar a influência da heterozigose (diferença dos cruzados em relação à média dos pais) no ganho de peso pré-desmame (GNDa) de terneiros Pampiano-
Braford, procurando estabelecer quanto da heterose se deve à heterozigose materna e quanto à individual, em animais criados no ambiente de produção do Rio Grande do Sul; comparar as diferentes composições raciais, associando os valores aditivos dos animais e os valores devidos aos diferentes graus de heterozigose. Por outro lado, fez-se uma investigação sobre os fatores de correção para efeitos ambientais conhecidos e predizíveis que estavam sendo aplicados aos dados no modelo empregado, e procurou-se estabelecer procedimentos de análise estatística flexíveis aos diferentes conjuntos de dados, para estudo dos efeitos da heterozigose em rebanhos cruzados.

\section{MATERIAL E MÉTODOS}

Foram analisados os dados de produção do Grupo DeltaG, que cria animais das raças Hereford e Pampiano-Braford, no Rio Grande do Sul, Brasil, coletados no período entre 1985 e 1994. Foram avaliadas informações de 4186 animais. A tabela 1 apresenta a distribuição das diferentes composições de heterozigose da vaca e do produto, no arquivo avaliado.

Como as características pré-desmame são grandemente influenciadas pela produção de leite da vaca, a heterose foi dividida em duas partes: 1) individual, referente à expressão do genótipo do terneiro para crescimento e adaptação; 2) materna, que se refere à expressão do genótipo da mãe cruzada, em termos de produção de leite, e outros componentes maternos. A heterose foi estimada após corrigir o GNDa para os efeitos ambientais conhecidos e predizíveis: idade da vaca, data juliana de nascimento e idade do terneiro ao desmame (pré-correções propostas por FRIES, (1984); também levou-se em conta os valores genéticos aditivos do touro, da vaca e do produto, além do efeito ambiental contido no grupo contemporâneo. Esses componentes do peso

Tabela 1 - Distribuição dos dados conforme a composição das heterozigoses indiviual e materna.

\begin{tabular}{ccccc}
\hline \multicolumn{5}{c}{$\begin{array}{c}\text { HETEROZIGOSE } \\
\text { MATERNA }\end{array}$} \\
$\begin{array}{c}\text { HETEROZIGOSE } \\
\text { INDIVIDUAL }\end{array}$ & 0.0000 & 0.5000 & 1.0000 & TOTAL \\
& & & & \\
\hline 0.0000 & 777 & - & - & 777 \\
0.5000 & 190 & 426 & 2227 & 2843 \\
1.0000 & 566 & - & - & 566 \\
& & & & \\
\hline & 1533 & 426 & 2227 & 4186
\end{tabular}


ao desmame foram estimados pelo Método GenSys (SCHENKEL \& BRITO, 1994). Após obtidas estas estimativas e corrigido o peso ao desmame, a heterose foi estimada através do Programa Estatístico Givens (FRIES, 1987).

O modelo operacional utilizado foi:

$$
\mathbf{Y a}=\mathbf{G C}+\mathbf{T}+\mathrm{V}+\mathrm{SM}+\beta_{1} \mathrm{HzM}+\beta_{2} \mathrm{HzI}+\mathbf{e}
$$

onde: $\quad$ Ya $=$ Resposta pré-corrigida para efeitos ambientais conhecidos (Ac); GC = Grupo Contemporâneo; $\mathrm{T}$ = Efeito genético aditivo do touro; $\mathrm{V}=$ Efeito genético aditivo da vaca; $\mathrm{SM}=$ Segregação Mendeliana; $\beta_{1}$ e $\beta_{2}=$ coeficientes de regressão; HzI $=$ heterozigose individual; $\mathrm{HzM}=$ heterozigose materna.

A estimação dos efeitos contidos no modelo foi feita em duas etapas distintas, através de métodos diretos e indiretos, de forma interativa:

$$
\begin{aligned}
\mathbf{Y a}= & \mathbf{G C}+\mathbf{T}+\mathbf{V}+\mathbf{S M} \\
& +\beta_{1} \mathbf{H z M}+\beta_{2} \mathbf{H z I}
\end{aligned}
$$

Para acelerar o processo interativo acima descrito, empregou-se a Técnica de Aproximação pelo Intercepto Comum (SCHAEFFER \& MAO, 1987). Também foi utilizado o Teste da Falta de Ajustamento dos Dados, através do F-teste (WEISBERG, 1980), com a finalidade de verificar se a relação entre a resposta e as variáveis contínuas contidas no modelo era linear. As heterozigoses materna e individual foram calculadas levando-se em conta a proposição feita por BÉRTOLI (1994), onde:

$$
\mathrm{HzZT}=\sum_{\mathrm{j}=1}^{\mathrm{z}} \mathrm{CRT}_{\mathrm{j}}+\sum_{\mathrm{j}=1}^{\mathrm{z}} \mathrm{CRV}_{\mathrm{j}}-2\left(\sum_{\mathrm{j}=1}^{\mathrm{z}} \mathrm{CRT}_{\mathrm{j}} \times \sum_{\mathrm{j}=1}^{\mathrm{z}} \mathrm{CRV}_{\mathrm{j}}\right)
$$

sendo: $\mathrm{HzZT}=$ heterozigose Zebu-Taurino; $\mathrm{z}=\mathrm{n}^{\circ}$. raças Zebuínas.

$$
\mathrm{HzZZ}=\left(\sum_{\mathrm{j}=1}^{\mathrm{z}} \mathrm{CRT}_{\mathrm{j}} \times \sum_{\mathrm{j}=1}^{\mathrm{z}} \mathrm{CRV}_{\mathrm{j}}\right)-\sum_{\mathrm{j}=1}^{\mathrm{z}}\left(\mathrm{CRT}_{\mathrm{j}} \times \mathrm{CRV}_{\mathrm{j}}\right)
$$

sendo: $\mathrm{HzZZ}=$ heterozigose Zebu-Zebu.

$$
\mathrm{HzTT}=\left(\sum_{\mathrm{i}=1}^{\mathrm{t}} \mathrm{CRT}_{\mathrm{i}} \times \sum_{\mathrm{i}=1}^{\mathrm{t}} \mathrm{CRV}_{\mathrm{i}}\right)-\sum_{\mathrm{i}=1}^{\mathrm{t}}\left(\mathrm{CRT}_{\mathrm{i}} \times \mathrm{CRV}_{\mathrm{i}}\right),
$$

sendo: HzTT $=$ heterozigose Taurino - Taurino. $\mathrm{t}=\mathrm{n}^{\circ}$. raças Taurinas.

\section{RESULTADOS E DISCUSSÃO}

Os resultados encontrados indicaram efeito positivo e estatisticamente significativo $(\mathrm{P}<0,01)$ da heterozigose individual e materna no GNDa. A tabela 2 apresenta os valores estimados para os regressores de heterozigose individual e materna. Estes valores referem-se à média dos produtos com heterozigose máxima, ou seja, os terneiros $F_{1}$ (Nelore x Hereford) têm 9,06\% do seu GNDa devido à heterozigose individual e as vacas $\mathrm{F}_{1}(\mathrm{Ne}-$ lore x Hereford) são responsáveis por $20 \%$ do GNDa dos seus produtos. Cabe salientar que os valores da heterose materna estimados neste estudo estão um pouco acima da média encontrada na literatura. Entre as possíveis causas que interferiram na estimação da heterose, considerou-se que o grande número de reprodutores múltiplos utilizados neste conjunto de dados (cerca de $90 \%$ dos terneiros não possuem pai conhecido) deve ter contribuído em grande parte nas dificuldades em obter-se resultados mais precisos.

Os resultados das análises feitas sobre as pré-correções para idade da vaca, data juliana de nascimento e idade do terneiro ao desmame, que foram aplicadas ao conjunto de dados estudado denotaram que existe uma defasagem nos valores que estavam sendo utilizados, embora tenha sido considerado a necessidade de um estudo mais detalhado do modelo operacional empregado nas análises. CAMPOS et al. (1988) também observaram resíduos dos efeitos ambientais em dados pré-corrigidos, tendo salientado a importância de se estimar de forma precisa estes valores, pois os mesmos interferem de forma direta nos programas de avaliação genética. Mesmo obtendo valores específicos para os diferentes conjuntos de dados, ROSO (1995) indica a reestimação destes valores, sempre que possível.

De posse dos valores médios da heterose do conjunto de dados avaliado foram feitos cálculos para quantificar o incremento da produção advindo

Tabela 2 - Estimativas encontradas para os regressores de heterozigose individual (HzI) e materna (HzM) no ganho de peso pré-desmame (GNDa).

VALOR ESTIMADO

COVARIÁVEL

$(\mathrm{kg})$

$(\%)$

\begin{tabular}{lrr}
\hline & & \\
HzI & 13,79 & 9,06 \\
HzM & 34,08 & 20,00 \\
& & \\
\hline
\end{tabular}


do uso do cruzamento. Considerando-se a heterose total de $26,35 \mathrm{~kg}$, e a média do ganho de peso prédesmame ajustado de $164,81 \mathrm{~kg}$, observamos que houve um incremento de $15,99 \%$ em quilogramas de terneiro desmamado devido à heterose. CUNDIFF $\boldsymbol{e t}$ al. (1974) encontraram um incremento através da heterose de $23 \%$ na quantidade de quilogramas de terneiros desmamados por vaca exposta à reprodução. GREGORY \& CUNDIFF (1980) estudaram o efeito da heterose sobre a taxa de crescimento dos terneiros e concluíram que $60 \%$ ou mais da heterose deve-se às características maternas. Estes resultados vêm ao encontro dos obtidos no presente trabalho, no qual a heterose materna foi responsável por $71 \%$ do incremento no ganho de peso do nascimento ao desmame dos animais cruzados. O desempenho das vacas e dos terneiros cruzados foi calculado levando em conta o valor genético aditivo dos mesmos e a $\mathrm{HM}$ no primeiro caso e a HI no segundo.

Ao comparar o desempenho materno verificou-se que as vacas 1/2 sangue (Nelore x Hereford) desmamaram, em média, terneiros $32,21 \mathrm{~kg}$ mais pesados que os das vacas Hereford. Em segundo lugar, ficaram as vacas $1 / 4$ Nelore, que desmamaram terneiros $12,39 \mathrm{~kg}$ mais pesados que os das vacas Hereford. Esses resultados estão de acordo com o esperado, ou seja, as mães $1 / 2$ sangue obtiveram o melhor desempenho.

$\mathrm{Na}$ análise do desempenho pré-desmame das diferentes composições raciais dos terneiros, obteve-se a seguinte classificação: os terneiros cruzados superaram os Hereford, sendo que os $1 / 2$ sangue foram os mais pesados $(10,67 \mathrm{~kg}$ acima dos $\mathrm{He}$ reford), seguidos pelos $1 / 4$ Nelore, 6kg mais pesados, comparando com a média dos Hereford. Todavia, esses resultados não levam em conta a heterose materna que, conforme já citado anteriormente, exerce grande influência no desempenho dos terneiros ao desmame. Assim sendo, incluiu-se a HM no desempenho dos terneiros, de acordo com a composição racial das vacas. Como os valores de HM encontrados foram muito altos, houve mudança na classificação dos terneiros cruzados (tabela 3). Em virtude da estrutura, o conjunto de dados foi dividido em dois subconjuntos. A tabela 3 refere-se ao subconjunto 2, no qual não foram incluídos terneiros Hereford e 1/2 sangue. Analisando a tabela 3, também pode-se observar um menor valor genético aditivo dos produtos com maior composição racial zebuína. Entretanto, a diferença de até $6,72 \mathrm{~kg}$ entre
Tabela 3 - Desempenho dos terneiros ao desmame, conforme sua composição racial (CRP) e da vaca (CRV), valor genético (VGP),e as heteroses individual (HI) e materna (HM).

\begin{tabular}{cccccc}
\hline CRP & CRV & $\begin{array}{c}\text { VGP } \\
(\mathrm{kg})\end{array}$ & HI $(\mathrm{kg})$ & HM $(\mathrm{kg})$ & TOTAL $(\mathrm{kg})$ \\
\hline $3 / 8$ & $1 / 2$ & 1,23 & 6,90 & 34,08 & 42,21 \\
$1 / 4$ & $1 / 2$ & 0,48 & 6,90 & 34,08 & 41,46 \\
$1 / 2 \mathrm{~F}_{2}$ & $1 / 2$ & 0,04 & 6,90 & 34,08 & 41,02 \\
$3 / 8$ & $1 / 2 \mathrm{~F}_{2}$ & $-0,22$ & 6,90 & 17,04 & 23,72 \\
$1 / 4$ & $1 / 2 \mathrm{~F}_{2}$ & $-0,62$ & 6,90 & 17,04 & 23,32 \\
$3 / 8$ & $1 / 4$ & $-2,15$ & 6,90 & 17,04 & 21,79 \\
$3 / 4$ & $1 / 2 \mathrm{~F}_{2}$ & $-5,21$ & 6,90 & 17,04 & 18,73 \\
$1 / 4$ & $\mathrm{HER}$ & 1,51 & 6,90 & 0,00 & 8,41 \\
& & & & & \\
\hline
\end{tabular}

onde: $\mathrm{HER}=$ Hereford, e as frações correspondem à composição racial zebuína, no cruzamento com Hereford; $1 / 2 \quad F_{2}$ significa $1 / 2$ sangue de segunda geração.

os extremos de VGP, no caso os animais $1 / 4$ e 3/4, não foi capaz de suplantar os efeitos heteróticos no desempenho dos terneiros ao desmame.

\section{CONCLUSÃO}

A partir dos resultados obtidos, nas condições do presente trabalho, pode-se concluir que: a heterozigose individual e a materna influenciam significativamente o ganho de peso do nascimento ao desmame de terneiros Pampiano-Braford, de forma linear. Análises exploratórias indicam que as pré-correções para idade da vaca, idade do terneiro ao desmame e data juliana de nascimento podem não ajustar corretamente o ganho do nascimento ao desmame estudado. A estrutura e conexidade do conjunto de dados avaliado demonstram problemas para estimação da heterose. Finalmente, a heterozigose interfere na avaliação do valor genético dos animais cruzados, devendo, portanto, ser este efeito incluído nos modelos utilizados em programas de avaliação genética de bovinos de corte;

\section{REFERÊNCIAS BIBLIOGRÁFICAS}

BÉRTOLI, C.D.; SEVERO, J.L.P. Fatores de correção para efeitos ambientais utilizados pelo Gensys Consultores Associados. Porto Alegre: Gensys Consultores Associados, 1994. 9p .(trabalho não publicado - R. Guilherme Alves, 170/34, 90680-000 Porto Alegre, RS.).

CAMPOS, L.T., SILVA, P.R., FRIES, L.A. Fatores de correção que afetam o ganho do nascimento à desmama em bovinos da raça Nelore. In: CONGRESSO BRASILEIRO DE PESQUISA DE ZEBU, 1., 1988, Uberaba. Anais... Uberaba: Epamig, 1989. 475 p. p. $108-123$.

CHAGAS, E.C. Cruzamento planejado como alternativa de produção. In: JORNADA SOBRE PRODUÇÃO DE NOVILHO JOVEM. 1., 1983, Anais... Bagé:EMBRAPAUEPAE, 1983. 152 p. p. 75-93. 
CUNDIFF, L.V., GREGORY, K.E., SCHWULST, F.J., et al. Effects of heterosis on maternal performance and milk

CUNNINGHAM, E.P. Formulation of breeding plans for dairy cattle and dual purpose cattle. Revista Brasileira de Genética, v. 12, supl. 3, p. 81-94, 1989.

FRIES, L.A. A study of weaning weights on Hereford cattle in Rio Grande do Sul, Brazil. Ames, Iowa 1984. 317 p. Thesis (PhD Melhoramento Animal) - Iowa State University, 1984.

FRIES, L.A. Givens - um sistema de análises estatísticas de dados não-balanceados por microcomputador. In: REUNIÃO ANUAL DA SBZ, 1987, Belo Horizonte, MG. Resumos... Belo Horizonte: SBZ, 1987. 304 p. p. 299.

FRIES, L.A. Conexão Braford-DEP. Fundamentos técnicos para seleção e cruzamentos. Porto Alegre: Conexão BrafordDEP, 1994. Algumas considerações sobre heterozigose e heterose: p. 9-13.

FRIES, L.A. Maximizar heterozigose ou heterose? In: SIMPÓSIO NACIONAL DE MELHORAMENTO ANIMAL. Ribeirão Preto,SP., 1996. Anais... Viçosa: Sociedade Brasileira de Melhoramento Animal, 1996. 2 p. (no prelo).

GREGORY, K.E., CUNDIFF, L.V. Crossbreeding in beef cattle: evaluation of systems Journal of Animal Science, v. 51, p. 1224-1242, 1980

KOGER, M. Effective crossbreeding systems utilizing zebu cattle.. Journal of Animal Science, v. 50, p. 1215-1220, 1980 .

LEAL, J.J.B. Cruzamentos sistemáticos e utilização de raças sintéticas. In: CONGRESO INTERNACIONAL DE CRUZAMIENTOS CON RAZAS CEBUINAS. 1., 1993 Rivera.,Uruguay Resumos... Rivera: Sociedad de Criadores de Cebu del Uruguay, 1993. 68 p. p. 63-68.

PADILHA, S.F. Conexão Braford-DEP. Fundamentos técnicos para seleção e cruzamentos. Porto Alegre: Conexão BrafordDEP., 1994. Como produzir Braford-DEP: p. 1-8.

ROSO, V.M. Fatores de correção para o ganho de peso médio diário de suínos da raça Landrace e sua influência na seleção. Porto Alegre: GenSys Consultores Associados, 1995. 17p. (trabalho não publicado - R. Guilherme Alves, 170/34, 90680-000 Porto Alegre, RS.)

SCHAEFFER, L.R., MAO, I.L. Curso de genetica animal. Zaragoza, 1987. Notes on linear model theory, best linear unbiased prediction and variance component estimation: 237 p. (Apostila)

SCHENKEL, F.S., BRITO, F.V. The GenSys method for genetic evaluation of beef cattle. In: WORLD CONGRESS ON GENETICS APPLIED TO LIVESTOCK PRODUCTION, 3. 1994, Guelph. Proceedings... Guelph:Univesity of Guelph, 1994. 519 p. p. $192-195$.

WEISBERG, S. Applied linear regression. Ithaca, New York: John Wiley \& Sons, 1980. 203 p. production in Hereford, Angus and Shorthorn cattle. Journal of Animal Science, v. 38, n. 4, p. 728-745, 1974. 